\title{
Educación y gestualidad: inquietudes estéticas en torno al performance de los profesores en el escenario escolar
}

\section{Artículo de investigación}

\section{Andrés Felipe Álvarez Rivera}

Universidad de San Buenaventura, Medellín, Colombia

afelipealvarez@gmail.com

\section{Edilberto Hernández González}

Universidad de San Buenaventura, Medellín, Colombia. edilberto.hernandez@usbmed.edu.co

Recibido: 24 de octubre de 2018

Aprobado: 25 de julio de 2019

Cómo citar este artículo: Álvarez Rivera, Andrés Felipe y Edilberto Hernández González (2020). Educación y gestualidad: inquietudes estéticas en torno al performance de los profesores en el escenario escolar. Calle 14: revista de investigación en el campo del arte 15(28). pp. 354-367.

https://doi.org/10.14483/21450706.16277

Este artículo fue presentado como requisito para optar por el título de Magister en Ciencias de la Educación, en la Universidad de San Buenaventura, sede Medellín. Gracias al Dr. Edilberto Hernández González, asesor del trabajo de investigación y a Liliana Franco Cano, quien realizó aportes a este artículo para su publicación.

\section{(2) (1)}

https://creativecommons.org/licenses/by/4.0/deed.es 


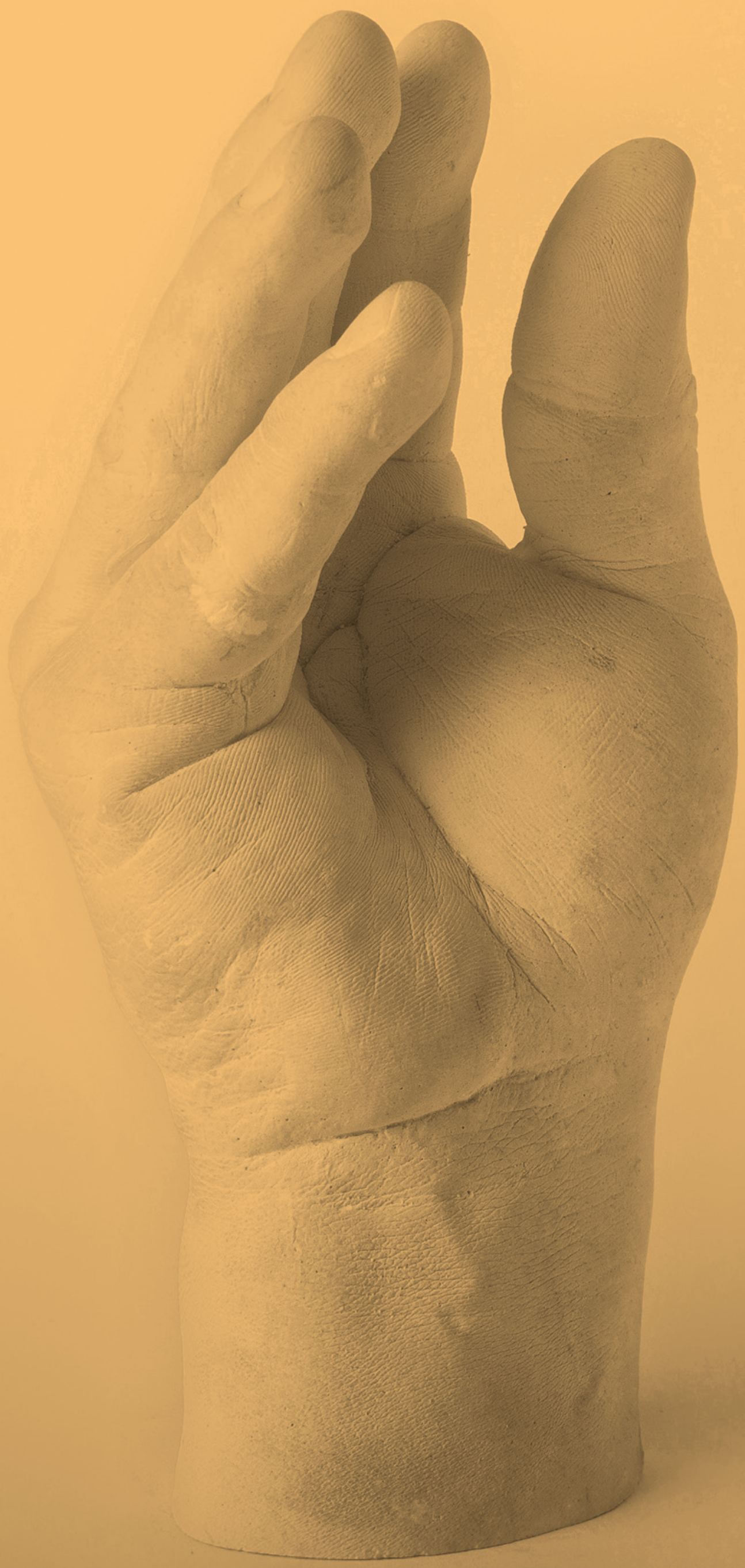


Educación y gestualidad: inquietudes estéticas en torno al performance de los profesores en el escenario escolar

\author{
Resumen \\ El artículo es derivado de la investigación Educación y gestualidad: una aproximación al \\ performance de los maestros en el escenario del aula escolar, la cual se interesó por comprender \\ las coreografías gestuales de las manos de los profesores en tanto acciones performativas, \\ educativas y estéticas. El estudio fue concebido teórica y metodológicamente desde la \\ perspectiva de la Investigación Basada en las Artes - IBA, implementando la experimentación \\ con artes como método para articular la teoría con los procesos de comprensión y creación. \\ Esto derivó en un proyecto de creación en el marco de una residencia artística, en el cual \\ se materializaron las comprensiones en torno a la gestualidad corporal, a través de diversas \\ expresiones estéticas contemporáneas, otorgando a los gestos voces que les permitan ser \\ concebidos por fuera de los sentidos didácticos que tradicionalmente la pedagogía les ha \\ asignado.
}

\title{
Palabras clave
}

Coreografías gestuales; educación; gestualidad; investigación basada en artes; prácticas corporales.

Education and Gestuality: Aesthetic Concerns about the Performance of Teachers in a School Setting

\begin{abstract}
This article is derived from the research project Education and gestures: an approach to the performance of teachers in the classroom setting, aimed at understanding the gestural choreographies of teachers' hands as performative, educational and aesthetic actions. The study was conceived theoretically and methodologically from an Arts-Based Research approach, implementing experimentation in arts as a method of articulating theory with the processes of understanding and creation. This led to a creative project within the framework of an artistic residency, which materialized a deep understanding about body gestures through various contemporary aesthetic expressions, giving voices to gestures in order to allow them to be conceived outside of the didactic senses that pedagogy has traditionally assigned to them.
\end{abstract}

\section{Keywords}

Gestural choreography; education; gestuality; arts-based research; body practices.

Éducation et gestualité : préoccupations esthétiques concernant la performance des enseignants dans la scène scolaire

\section{Résumé}

L'article est dérivé du projet de recherche Education et gestualité : une approche de la performance des enseignants dans la salle de classe, visant à comprendre les chorégraphies gestuelles des mains des enseignants comme des actions performatives, éducatives et esthétiques. L'étude a été conçue théoriquement et méthodologiquement à partir d'une approche de recherche basée sur les arts, mettant en œuvre l'expérimentation dans les arts comme une méthode d'articulation de la théorie avec les processus de compréhension et de création. Cela a conduit à un projet créatif dans le cadre d'une résidence artistique, qui a matérialisé une compréhension profonde des gestes corporels à travers diverses expressions esthétiques contemporaines, donnant ainsi des voix aux gestes afin de leur permettre d'être conçus en dehors des sens didactiques que la pédagogie leur attribue traditionnellement. 


\section{Mots clés}

Chorégraphie gestuelle ; éducation ; gestualité ; recherche basée sur les arts ; pratiques corporelles.

Educação e gestualidade: inquietudes estéticas ao redor da performance dos professores no cenáric escolar

\section{Resumo}

O artigo é derivado da investigação Educação e gestualidade: uma aproximação à performance dos professores no cenário de sala de aula escolar; na qual se interessou por compreender as coreografias gestuais das mãos dos professores como ações performativas, educativas e estéticas. $O$ estudo foi concebido teórica e metodologicamente a partir da perspectiva da Investigação Baseada na Artes- IBA, implementando a experimentação com artes como método para articular a teoria com os processos de compreensão e criação. Isto levou a um projeto de criação no âmbito de uma residência artística, no qual se materializaram as compreensões ao redor da gestualidade corporal, através de diversas expressões estéticas contemporâneas, atribuindo aos gestos vozes que Ihes permitam ser concebidos por fora dos sentidos didáticos que tradicionalmente a pedagogia Ihes atribui.

\section{Palavras chave}

Coreografias gestuais; Educação; gestualidade; investigação baseada em artes; práticas corporais.

\section{Tachaci sumaglla tapuchispa ruraikunamanda, imami iurankuna iachachidurkuna}

\section{Maillallachiska}

Kai kilkai nikumi munakunsi kawachinga tupuchispa allilla imasami iachachidurkuna wawakunata mailla mailla parlanaku. Kai kansi llapa unaimanda sakiska sug kati samunakuskata iachachingapa kaipi munaku mana chingachu ruraikuna,parlukuna kawachinkuna kausai, chasallata ima munaska rimanaku nukanchi kikin iuachispa llukanchi kanga.

\section{Rimangapa Ministidukuna}

Kusikuspa kawachingapa; ruraikuna Nukanchipa nawiwa; iachaikui; ruraikuna;tapuchii ruraikunamanda iuiarispa; Nukanchipa Tukui llukaskawa rurai. 


\section{Introducción}

Actualmente se encuentran diversidad de estudios que centran la atención en la escisión y polarización sostenida en occidente con relación a la comprensión del ser humano. Revisiones como la de Scharagrodsky (2001), Sibilia (2005) y Le Breton (2006) dejan entrever cómo se constituyeron e instauraron, de forma hegemónica, una serie de dualismos en torno a una supuesta materialidad de la existencia humana, que comprende lo corporal, lo sensorial, la experiencia, y una inmaterialidad referida al espíritu, el pensamiento, la razón. Estas separaciones han sido ampliamente cuestionadas, al igual que la estigmatización de lo corporal como el lugar de lo corrupto, perecedero, irracional y pasional.

Scharagrodsky (2001) sostiene que las instituciones educativas fueron un medio imprescindible para la popularización de esa visión. Según este autor, la aparente preponderancia del enciclopedismo y racionalismo en nuestras escuelas, sumado a la especial importancia curricular que parece tener las disciplinas que cultivan el "espíritu" frente a aquellas que se ocupan del cuerpo, podría hacer pensar que la educación corporal ha sido desatendida; sin embargo, una mirada detenida muestra que el control regulado del cuerpo ha sido una de las claridades más arraigadas del discurso pedagógico moderno desde sus inicios. En este mismo sentido, Pedraza (2009) sitúa los vínculos entre "las comprensiones del cuerpo y los modelos escolares de educación [con las] pedagogías del conocimiento", para indicar que existe un "ordenamiento epistemológico hegemónico" (p. 49) en el que ciertas comprensiones del cuerpo y de lo sensible, dan relevancia a formas específicas de conocer y organizar la experiencia.

En los contextos contemporáneos, a la par con cierta persistencia de estas concepciones $y$, a veces en oposición, se evidencian nuevas y complejas configuraciones subjetivas de los cuerpos. En los contextos educativos, cada vez con mayor fuerza, emerge la invención de cuerpos que son en sí mismos su pensar. Estos cuerpos, un tanto osados, interrogan a esos cuerpos aun enajenados en dispositivos claramente homogenizadores. Los nuevos cuerpos reconocen la posibilidad de inventar sus propias formas de ser, de poner en cuestión las disciplinas escolares, de arriesgarse a decir y no solo de obedecer.

Así pues, el presente artículo procede de la investigación titulada "Educación y gestualidad: una aproximación al performance de los maestros en el escenario del aula escolar", la cual se desarrolló en el contexto de la Maestría en Educación de la Universidad de San Buenaventura, Medellín, Colombia. Este estudio se incorporó a las discusiones antes mencionadas, con el propósito de dar continuidad a las reflexiones sobre el papel del cuerpo en los escenarios educativos y materializar esas inquietudes en una propuesta estética. Particularmente, en este artículo se indaga el cuerpo como experiencia subjetiva en el contexto del aula escolar, centrándose en las coreografías gestuales de las manos de los profesores.

Esta investigación se concibió teórica y metodológicamente desde la perspectiva de la Investigación Basada en Artes - IBA, y de manera más puntual desde la experimentación con artes como modo de proceder en los procesos de búsqueda y creación. Para ello se partió de observaciones y registros audiovisuales de los cuerpos de los profesores en sus aulas de clases, siguiendo especialmente las coreografías gestuales de sus manos. Por su parte la fundamentación teórica se apoyó en una serie de planteamientos que posibilitaron situar y comprender el cuerpo en el contexto de la filosofía, la educación y el arte contemporáneo.

En la comprensión de los registros audiovisuales se tomó distancia respecto de algunos enfoques orientados a rastrear sus significados, como los conocidos diccionarios de gestos (Meo-Ziloo \& Mejía, 1983)ㄹ. El interés en este estudio fue la comprensión de los gestos en tanto acciones performativas, educativas y estéticas. La experimentación con los registros audiovisuales se realizó en el contexto de una residencia artística; $^{2}$ experiencia en la que se situaron diversas dimensiones de los gestos y se construyó una obra artística que se ocupó sensiblemente de las coreografías gestuales de las manos de los profesores para otorgarles otras voces, surgidas por fuera de los sentidos didácticos que tradicionalmente la pedagogía les ha asignado. La producción artística exploró también las posibilidades expresivas de diversas técnicas, materiales, formatos y configuraciones propias del arte contemporáneo, con el ánimo de invitar al público a participar de esos juegos coreográficos que acontecen en el aula como parte de la cotidianidad.

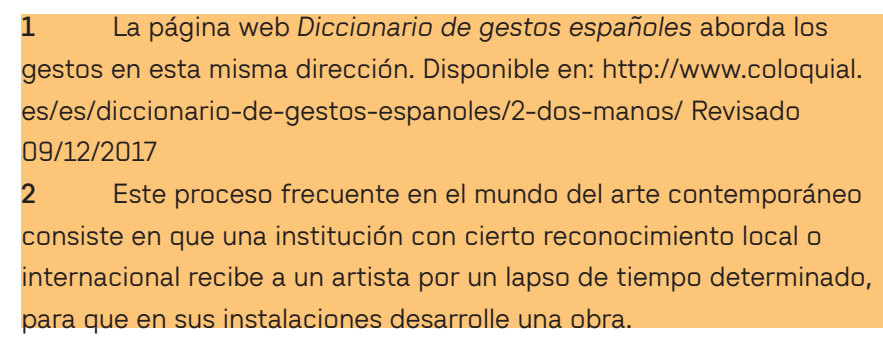




\section{La gestualidad corporal: encuadre teórico}

Vivir consiste en reducir constantemente el mundo al cuerpo, a través de lo simbólico que este encarna.

David Le Breton (2002)

Usualmente, cuando se hace referencia a lo educativo, se enfatiza en la relación que se teje en el encuentro de dos subjetividades que hacen presencia; estudiante y profesor. Sin embargo, hoy en día entendemos que lo educativo trasciende esta relación. En este orden de ideas, se quiere señalar que existen asuntos de lo educativo, con relación al profesor, que incluso anticipan la presencia de los estudiantes. Es decir, el profesor construye su subjetividad desde prácticas corporales específicas que inciden en lo educativo, que expresan -en tanto el profesor no puede renunciar a su cuerpo cuando está presente en el aula de clases-, lo cual remite a las palabras de Le Breton (2000) cuando afirma que "el encuentro del alumno con el educador, es también un encuentro [...] del educador consigo mismo" (p. 41).

Las prácticas corporales específicas aluden a eso que Beatriz Preciado (2002) señala como elementos del sistema que producen los cuerpos socialmente, en oposición a corporalidades o subjetividades dadas por generalidades atribuidas a supuestas leyes naturales 0 metafísicas. Se hace referencia entonces a las maneras de la sexualidad, las formas de vestir, de hablar, de moverse, de ser un cuerpo. Otros autores comparten este mismo horizonte de pensamiento. Sibilia (2005), por ejemplo, habla de la plasticidad del ser humano, y Serres (2009), considera que el cuerpo se erige a partir de realidades virtuales y no de realidades positivas; que este se construye a partir de la posibilidad de ser y no de una realidad dada, externa a la propia subjetividad. David Le Breton por su parte, argumenta que:

los gestos [...] no provienen de una fisiología pura y simple, ni de una única psicología, una y otra se superponen a una simbología corporal que les da sentido, se nutren de una cultura afectiva que cada sujeto vive a su manera (Le Breton, 2000, p. 36).

Así pues, se insiste en que la construcción de la existencia es una experiencia encarnada, de manera que un proyecto de subjetivación no puede desarrollarse más que en el cuerpo. La corporalidad es el espacio de producción subjetiva (Hernández, 2015), en tensión con la cultura y, a partir de prácticas específicas, como insiste Preciado (2002).
Comprender el cuerpo como experiencia subjetiva, suscita preguntas importantes sobre los vínculos cuerpo-aprendizaje: ¿Cómo se leen estas prácticas específicas en el contexto del aula de clase? ¿La educación puede tener sentido desde la idea de aprendizaje solamente en el cuerpo del profesor? Es decir, ¿se puede hablar de lo educativo en el cuerpo del profesor aun cuando no se sabe si hay aprendizaje en los estudiantes? ¿Qué transformaciones se producen en los cuerpos a partir de la experiencia de producción de nuevos conocimientos?

Al respecto de estas inquietudes, Hernández (2015), señala que las prácticas corporales son modos contemporáneos de subjetivación, en tanto prácticas performativas. En este contexto, lo performativo se entiende como esa condición de los cuerpos de encarnar los discursos y de decir de sí mismos (Hernández, 2015). Así, los performances de los profesores, al ser interrogados estéticamente, dejaron entrever otros pliegues de sus propias construcciones subjetivas y de sus maneras de producir conocimientos. La experiencia en el mundo está determinada por el cuerpo y la subjetividad es una producción que resulta de los flujos constantes de dicha experiencia. Esto significa que las manos y sus gestos, como artefactos que permiten la incorporación de lo simbólico en el mundo, incorporan dicho mundo en la propia subjetividad.

El arquitecto y autor Juhani Pallasmaa (2006), cita al poeta alemán Rilke en su ensayo sobre el escultor Auguste Rodin para demostrar su intuición sobre las manos como las mismas creadoras de la existencia y del lenguaje:

Las manos son un organismo complicado, son un delta en el que desemboca una vida que viene de muy lejos, para verterse en el gran torrente de la acción. Hay una historia de las manos; tienen de hecho su propia cultura, su belleza; se les concede el derecho a tener su propio desarrollo, sus propios deseos, sentimientos, humores y caprichos (Rilke, 1903, citado en Pallasmaa, 2006, p. 58).

La mano que acaricia toma la forma de lo acariciado, la mano que toca se convierte de algún modo en lo tocado. A diferencia de ser el lugar donde se tiene contacto con el mundo, como si fueran una línea limítrofe, como si esa realidad preexistiera más allá de la subjetividad; las manos son el mundo mismo, y es en ese flujo constante donde se produce y son producidas las subjetividades. 
Michel Serres (2011), afirma que el cuerpo, por medio de metamorfosis gestuales y posturas móviles, recupera "una presencia y una función cognitiva propias" (p.77). Expresa esta idea de la posibilidad al hablar del escalador que, en contacto con la pared de la montaña, se transforma y sufre una metamorfosis en la que sus cuatro extremidades se hacen prensiles y todos sus veinte dedos se convierten en agarres seguros para el equilibrio.

En esta metamorfosis radica gran parte de la potencia del gesto, que permite el paso de la imaginación a la transformación de la realidad y la cultura. El aprendizaje es la constante subjetivación del mundo en el cuerpo; se asimila el mundo imitando, pero se lo transforma inventando. "Por la primera acción, la vida se mantiene y desarrolla; por la segunda, la cultura viene al mundo" (Serres, 2011, p. 114).

\section{Los gestos en escena: encuadre metodológico}

Para este estudio se adoptó como encuadre metodológico la Investigación Basada en Artes (en adelante IBA), la cual refiere a un conjunto de métodos que se relacionan con el giro narrativo en la investigación en ciencias sociales en los años 80 (Finley, 2015), y con el debate público académico sobre la investigación en las artes, que en el contexto europeo toma fuerza desde el inicio de este milenio como respuesta a las políticas gubernamentales de financiación a la investigación académica (Borgdorff, 2010).

Desde la década de los ochenta se han venido adoptando, de forma separada por diferentes grupos de investigación, metodologías híbridas en las que los métodos tradicionales de las ciencias sociales se apoyan en herramientas propias de las artes como la fotografía, la literatura, la poesía, la pintura, el performance y la puesta en escena, entre otras.

En la bibliografía actual se encuentran referencias y definiciones bastante similares entre Metodologías Artísticas de Investigación en Educación e IBA. Para Marín (2011), las Metodologías Artísticas de Investigación en Educación, surgen como una manera de "aproximar los usos y tradiciones profesionales de la creación artística a las normas y criterios de la investigación en ciencias humanas y sociales" (p. 280). La IBA, por su parte, es descrita por Hernández (2008) como:
[...] un tipo de investigación de orientación cualitativa con procedimientos artísticos (literarios, visuales y performáticos) para dar cuenta de prácticas de experiencia en las que tanto los diferentes sujetos (investigador, lector, colaborador) como las interpretaciones sobre sus experiencias develan aspectos que no se hacen visibles en otro tipo de investigación (p.92).

De este modo, la perspectiva que Hernández propone de la IBA fue un horizonte prometedor en los procesos de concepción, diseño y comprensión de la investigación, ya que en ésta se privilegia la experiencia, lo que en otras perspectivas se da por hecho o se torna irrelevante; más cuando se pretendió acercar a cuestiones tan sutiles como los gestos corporales. La experiencia como fuente de conocimiento (no solo de la observación) y el arte como una forma genuina de experiencia (Eisner, 1998, como se citó en Hernández, 2008), invitan a seguir una lógica en la que la materialidad del lenguaje estético permite reflexionar sobre el cuerpo, teniéndolo a él mismo como medio.

En este sentido, la investigación que dio pie al presente artículo se desarrolló realizando articulaciones entre lo epistemológico y la experimentación con artes como forma de acercamiento y comprensión al performance de los profesores en sus aulas de clases. Las reflexiones sobre el cuerpo desembocaron en una exploración de la gestualidad de las manos, como médula de las prácticas performativas del cuerpo y en una lectura estética de ello con relación a lo educativo, para producir, finalmente, una obra artística, que permitió una experiencia reflexiva sobre dichos asuntos.

El trabajo exploratorio que se realizó desde la experimentación con arte se planteó en un doble pliegue. Por un lado, el trabajo audiovisual ayudó a tener una experiencia corporeizada de la investigación y preparó el terreno para reflexiones más conscientes del "aprendizaje y la enseñanza como experiencias corporeizadas" (Hernández, 2008, p. 110). De otro lado, se dio un proceso de indagación plástica constante a partir de la teoría, que sirvió como sustrato para la manera en que se relacionaron los distintos tipos de experiencias en la comprensión reflexiva.

En la investigación se reconocieron las coreografías gestuales de las manos de siete profesores en el desarrollo de sus actividades académicas en las aulas de clases, a quienes se les presentaron con antelación los propósitos de la investigación. Estos profesores hacían parte de 
dos instituciones diferentes, una de ellas correspondía al nivel de educación media y otra al universitario. Estos profesores desarrollaban clases de diversas áreas, entre ellas lengua extranjera, educación física, taller de diseño, español y literatura, y ciencias naturales.

Las coreografías gestuales de las manos de cada uno de estos profesores fueron grabadas en formato HD digital durante la hora clase, deteniendo la grabación siempre que el docente adoptara una posición corporal pasiva o repetitiva. Como condiciones de este sondeo, se utilizó un encuadre tipo americano que tomara con claridad los movimientos de las manos y brazos, a la vez que se aislaran otros gestos corporales. Se usó la técnica de cámara en mano con el fin de seguir las secuencias de movimientos del profesor y, aunque se grabó el audio, este no fue tenido en cuenta en el proceso de comprensión reflexiva.

El producto del trabajo audiovisual fue llevado a la residencia artística mencionada anteriormente, la cual se articuló al proceso de investigación. En ese contexto se realizó, durante un mes, una revisión del material audiovisual mencionado, proceso para el cual se hizo necesario afinar previamente la comprensión de los gestos de las manos a partir de la fundamentación teórica sobre el cuerpo, hasta concebirlos como la coreografía de los dedos y las palmas de las manos en un espacio-tiempo singular.

El material audiovisual fue editado a fin de reducirlo a un máximo de quince minutos de video por cada profesor. La edición implicó dejar por fuera los gestos repetitivos y los tiempos en los que el movimiento se tornaba imperceptible. Seguidamente se realizó una segunda edición en la que se hizo un pietaje por categorías, de manera que la totalidad de los gestos grabados fueron organizados en cuatro categorías de comprensión, a saber: 1. Secuencias y coreografías de los gestos de las manos; 2. Trayectorias dibujadas por los gestos de las manos en el espacio; 3. Formas que adoptan los gestos de las manos de los profesores; y 4 . Formas que los gestos de las manos de los profesores modelan en el espacio. A partir de estas categorías se desarrolló el proceso de formalización ${ }^{3}$ de las propuestas, que se materializaron en bocetos y posteriores pruebas para la producción artística.
En una segunda etapa de la residencia artística se llevaron a cabo escritos iniciales sobre algunas de las categorías y bocetos en dibujo; los que además de una exploración formal, tenían indagaciones sobre los materiales susceptibles de ser usados en las pruebas escultóricas. A continuación, se hicieron pruebas específicas de dibujo, video, audio y vaciados escultóricos con diferentes materiales. Finalmente, se construyeron algunas obras catalogadas como pruebas de autor ${ }^{4}$, con las cuales se conformó una muestra artística pública en la que se expusieron los resultados de la investigación y se efectuaron dos conversatorios académicos con estudiantes de la Licenciatura en Educación Artística de la Universidad de San Buenaventura, Medellín.

\section{El performance de la gestualidad: comprensión reflexiva e invención-creación}

La comprensión del material audiovisual y el conjunto de las experimentaciones en el contexto de la residencia artística constituyeron en sí mismos una experiencia sensible. Experiencia comprensiva, que si bien se sitúa metódicamente en un momento particular, ya estaba presente en la manera de concebir la investigación, en la construcción de los registros audiovisuales y en ese corpus sensible que no acaba de corporizarse en los productos de invención-creación, y que permanece moviendo el pensamiento y resonando en cada una de las reflexiones derivadas de la investigación.

Así pues, la comprensión reflexiva que orientó el proceder de la investigación no estuvo dirigido a indagar o descifrar los significados de los gestos de los profesores, tampoco a valorar sus consecuencias en el plano de los aprendizajes de los estudiantes. El proceder se encaminó, más bien, a comprender el desenvolvimiento subjetivo de las coreografías gestuales de las manos de los profesores, desde sus formas singulares de transcurrir en la cotidianidad del aula, donde el performance gestual fluye permanentemente. Siguiendo este proceder, los performances gestuales situados en los materiales audiovisuales se organizaron en las siguientes dimensiones. 


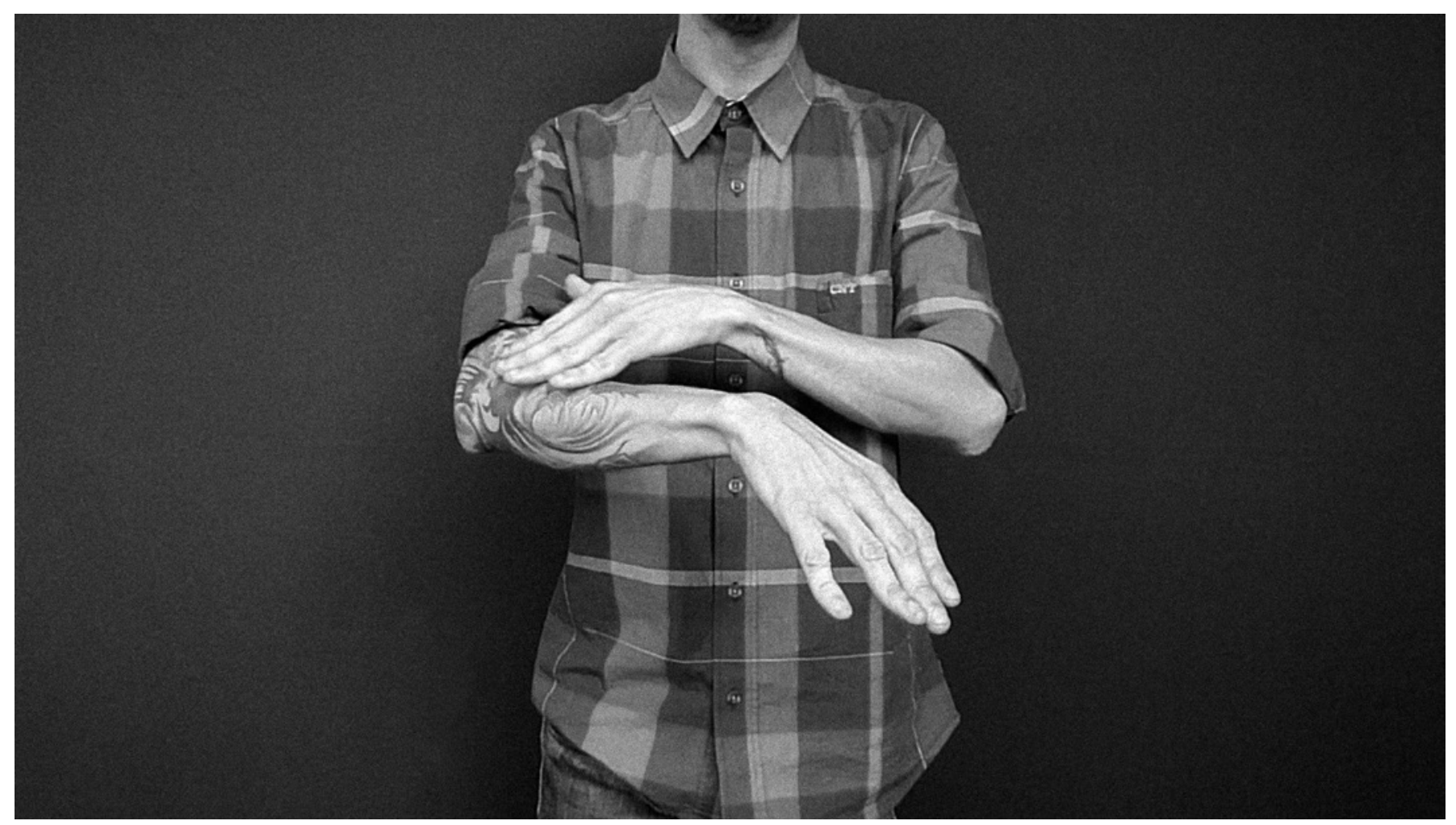

Imagen 1. Transducciones. (Álvarez, 2017). Imagen extraída del video para la video instalación. Dimensiones variables.

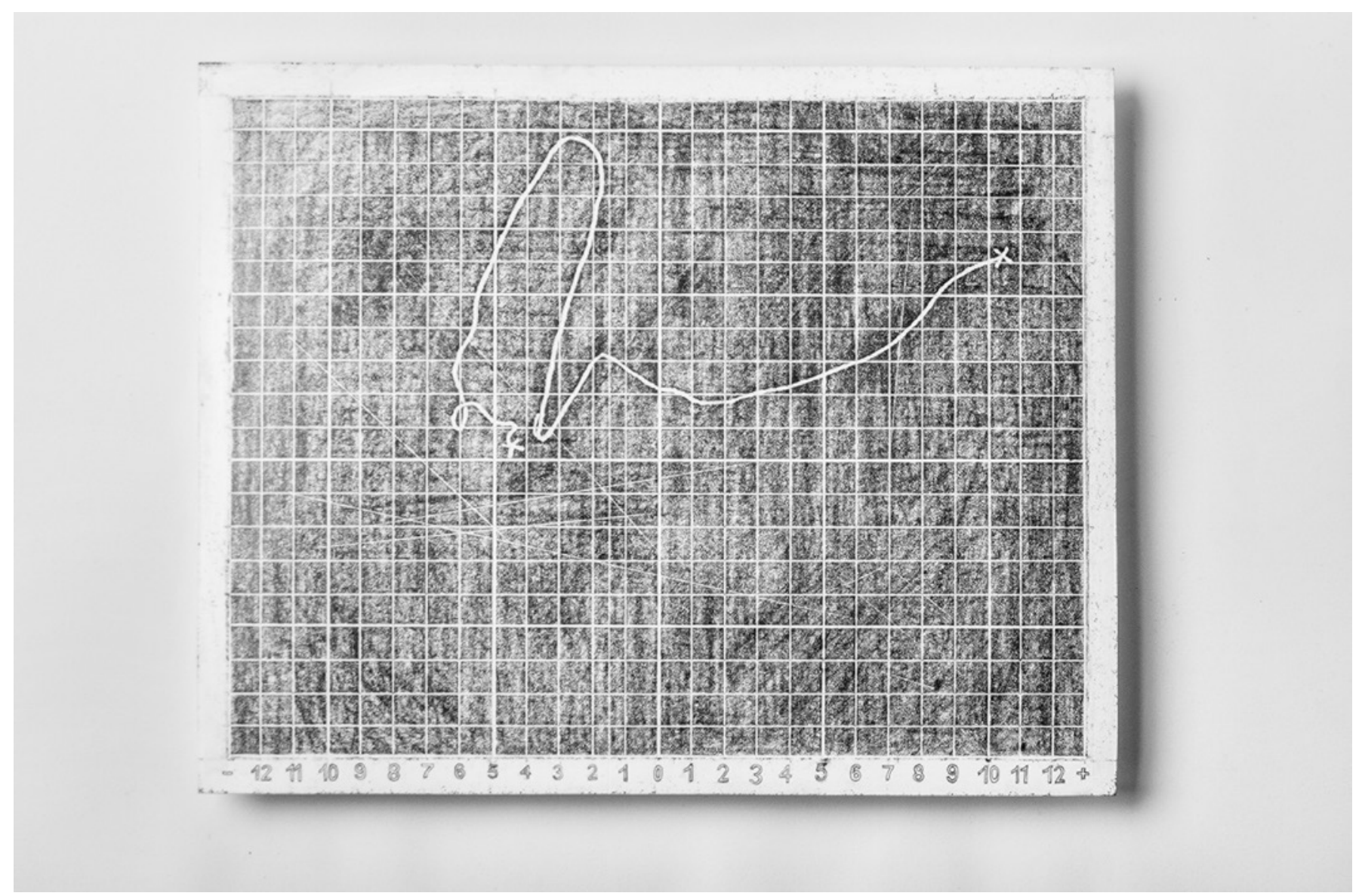

Imagen 2. Trayectoria \#1. (Álvarez, 2017). Prueba de artista. Grafito sobre papel. 29x21cm. Fotografía: Felipe Kardona. 


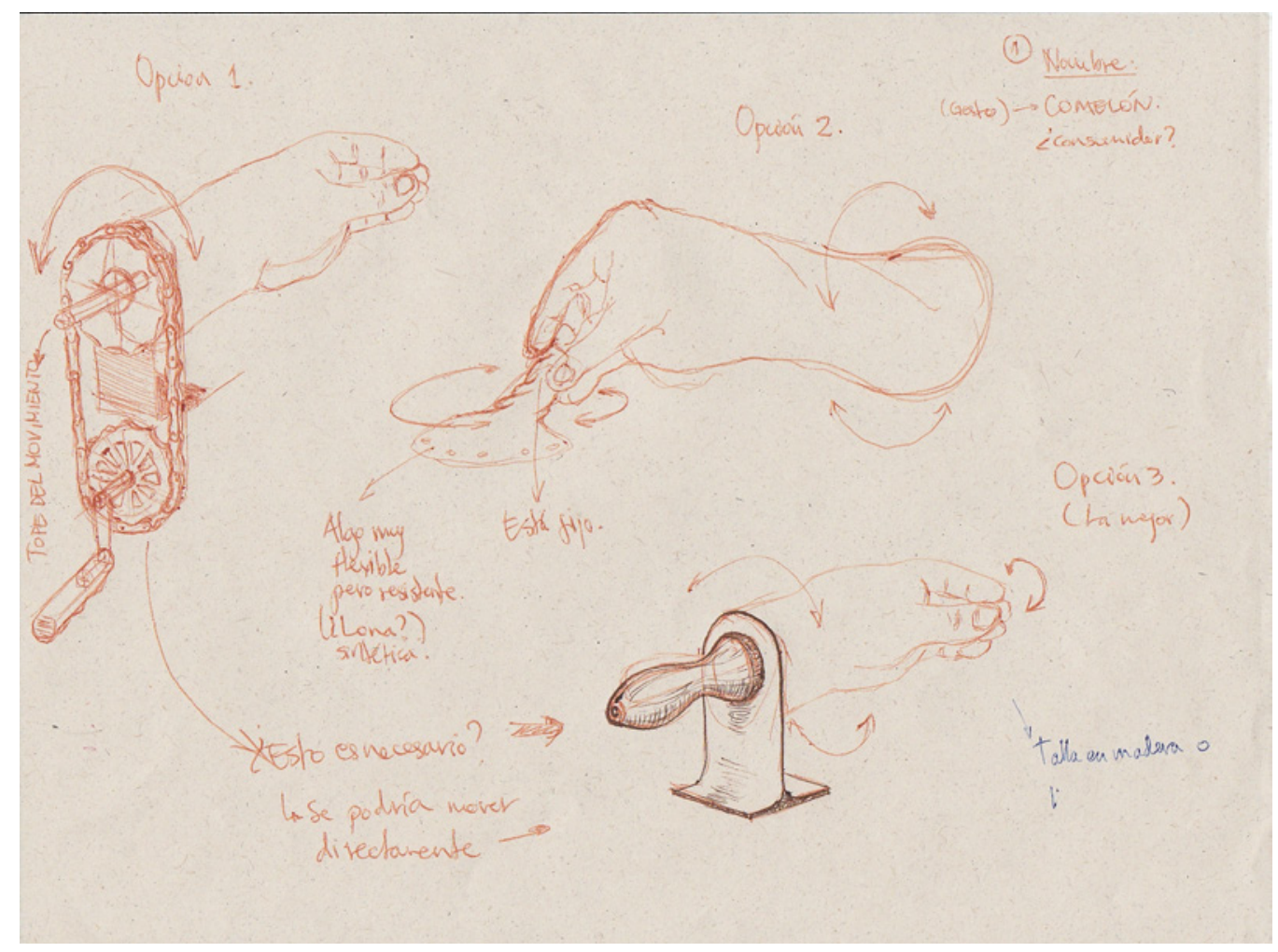

Imagen 3. Bocetos. (Álvarez, 2017). Tinta sobre papel. 29×21cm.

\section{Secuencias y coreografías de los gestos de las manos}

En esta dimensión se identificaron y describieron los movimientos de los gestos de las manos de los profesores de forma poética y exhaustiva. Dichas descripciones se agruparon de manera que propusieran una lectura fluida del movimiento. Como resultado de esta experimentación se generaron siete fragmentos, el siguiente es un ejemplo de los textos producidos en esta categoría:

El gesto se mece con el movimiento del cuerpo. La mano está tensa, el gesto muerto. No hay movimiento, solo la gravedad afecta el gesto. La mano se abre y florece. Uno de los dedos se mueve en círculos tocando el clítoris de la flor que era hace un momento. El gesto vuelve el interior en exterior y convierte el abajo en arriba. La mano roza con sensualidad el horizonte y se pavonea subiendo y bajando de manera cadenciosa. El dedo índice indaga en la feminidad del plano (Álvarez, 2017).

Esta serie de fragmentos fueron el soporte de dos formalizaciones artísticas. La primera, llamada Transducciones, en la cual el texto representa un estímulo que es reinterpretado corporalmente para producir una video instalación. La segunda, Ilamada
Coreografías, en la que una voz grave y profunda narra los movimientos de las manos y sus gestos, invitando al espectador a escenificar lo que va escuchando.

\section{Trayectorias dibujadas por los gestos de las manos en el espacio}

En esta segunda dimensión se rastrearon las trayectorias de los gestos de las manos de los profesores a través de la marcación de puntos sucesivos, directamente sobre la pantalla del monitor en el que son proyectadas. Lo anterior se realizó poniendo un papel transparente sobre el cual se marcaron, cuadro a cuadro, los trayectos recorridos por las manos en el espacio vacío. La materialización de esta exploración fue una serie de seis dibujos de gran formato.

\section{Formas que adoptan los gestos de las manos de los profesores}

En esta tercera dimensión se rastrearon gestos que no poseen una significación obvia. Los gestos hallados fueron descritos para dotarlos de forma y asignarles una intencionalidad. Por ejemplo, Gesto comelón: este gesto es una forma casi animal, los dedos agrupados en punta forman la boca que consume algo haciéndole torsión. Estas descripciones de los gestos se acompañan de bocetos de su posible formalización artística. 


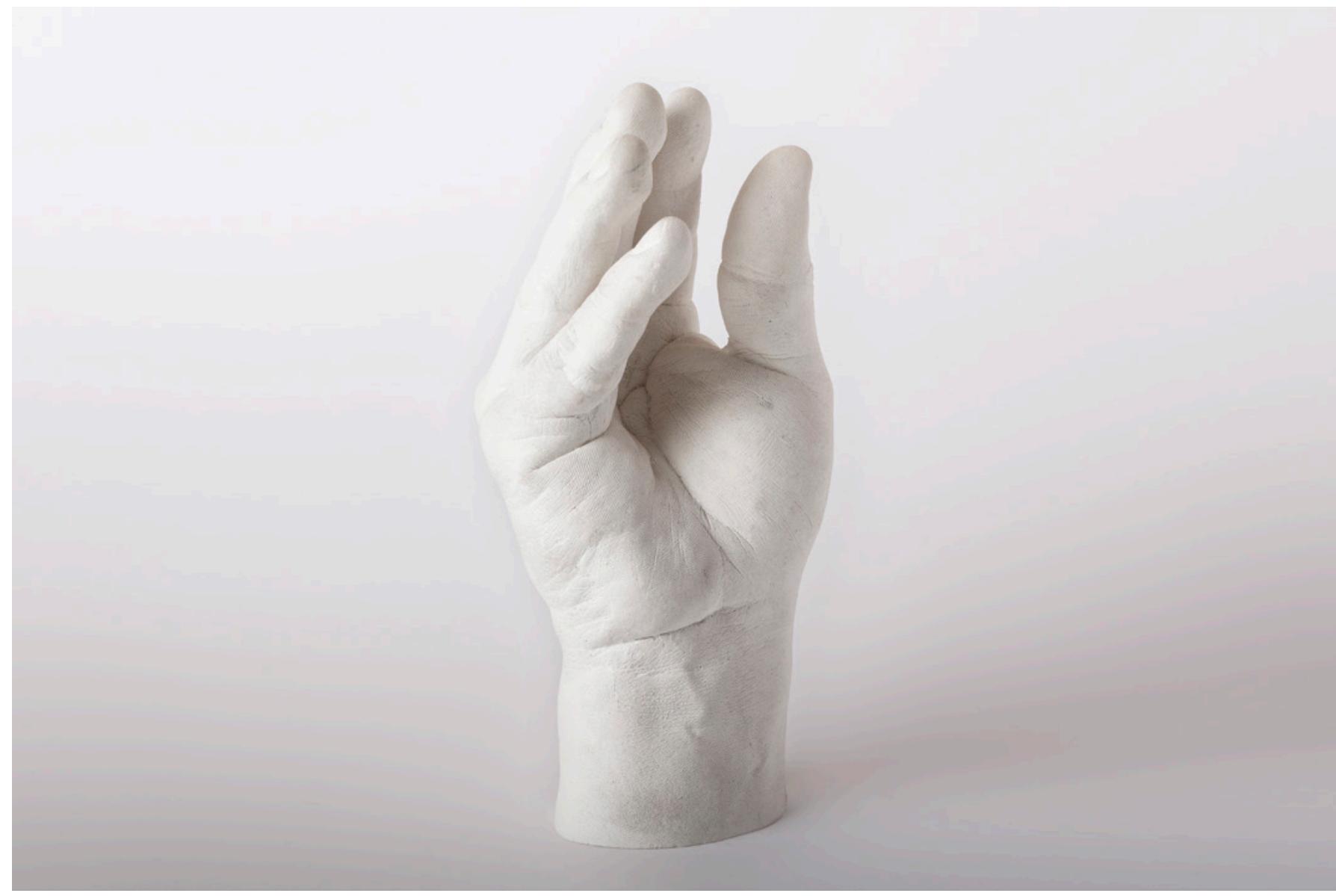

Imagen 4. Gesto \#1: Comelón. (Álvarez, 2017). Vaciado en yeso. 18×9×7,5cm. Fotografía: Felipe Kardona.

La exploración formal inicial se realizó con vaciados de yeso y concreto.

\section{Formas que los gestos de las manos de los profesores modelan en el espacio}

En esta última dimensión se describieron objetos imaginarios modelados en el espacio vacío a partir de los gestos de las manos de los profesores, durante el desarrollo de sus clases. La descripción de los gestos comprendió su forma, los materiales en que parecen estar hechos y las relaciones que establecen con las dimensiones corporales. Por ejemplo, el gesto agarra una bolsa de unos $30 \mathrm{~cm}$ de ancho a la altura del pecho, que se puede coger desde abajo y sopesar. La bolsa es densa como si tuviera agua.

Usando las descripciones mencionadas, se produjeron objetos en varias técnicas como el vaciado en yeso (bolsa de ideas sublimadas) o el modelado de barro rojo cocido (contenedor de ideas estimulantes), que permitieron dar otro cuerpo a la memoria del gesto que le había dado forma en la palabra. Esta materialización invita al espectador a encarnar dichos gestos. En la siguiente imagen se presenta una de las creaciones producidas en esta cuarta dimensión.

\section{Reflexiones finales}

A través del desarrollo de la investigación Educación y gestualidad: una aproximación al performance corporal de los maestros en el escenario del aula escolar, se pudo reconocer la fortaleza de la Investigación Basada en Artes (IBA) y la experimentación con artes como un proceder metódico potente para ampliar las posibilidades de la indagación-creación en el contexto de la investigación educativa.Estas articulaciones no solo permiten conjugar los modos de investigación en las artes y la investigación académica, sino que, además, se dinamizan las posibilidades comprensivas de la información a la vez que se reconfiguran los circuitos de creación artística.

Ahondando en la cuestión metodológica, se puede decir que la experimentación con artes, enriqueció y amplió las posibilidades de esta investigación desde las 


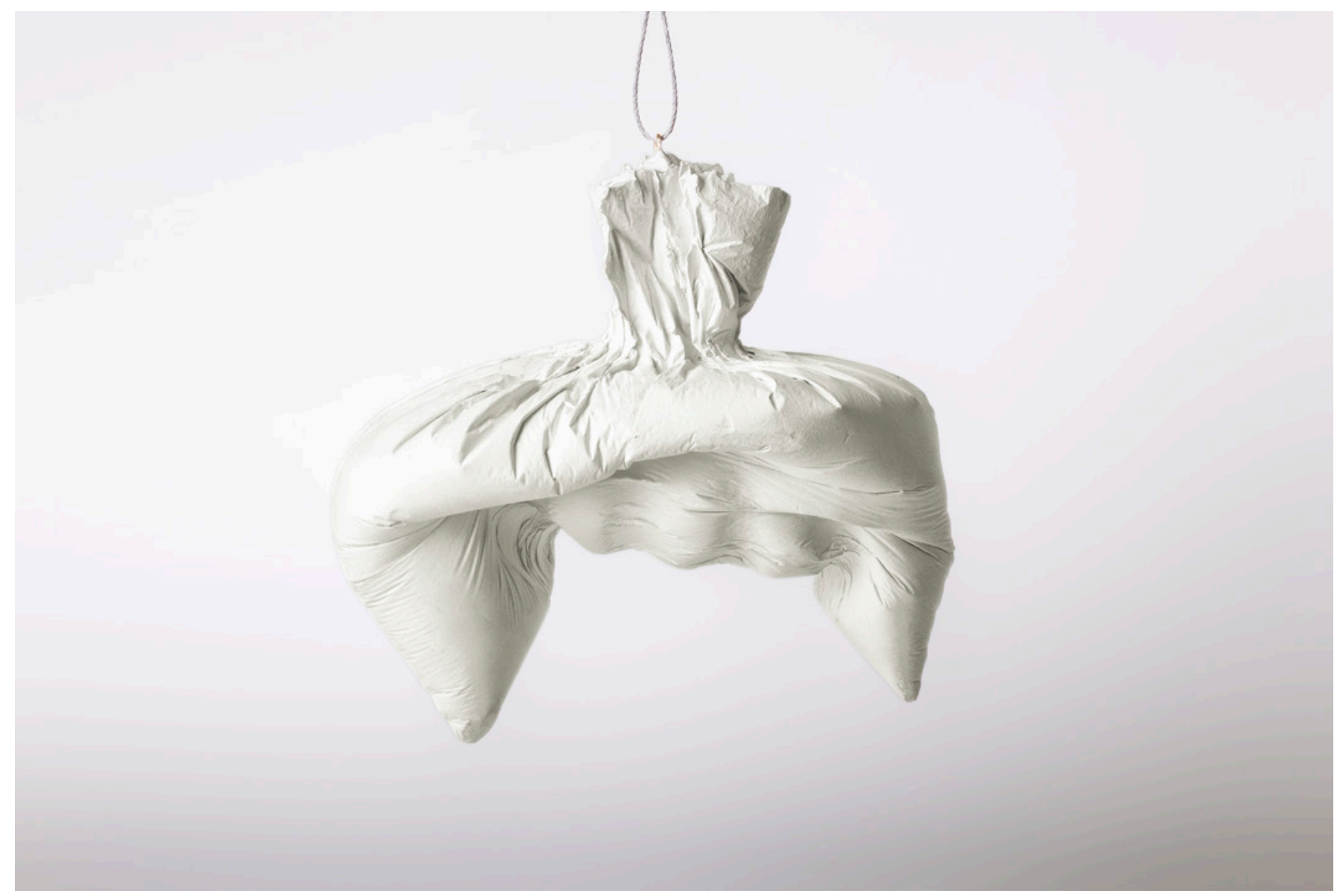

Imagen 5. Bolsa de ideas sublimadas. (Álvarez, 2017). Vaciado en yeso. 22×12×20cm. Fotografía: Felipe Kardona.

preguntas iniciales, hasta materializar estéticamente las construcciones epistémicas que se produjeron en el contexto de la práctica investigativa. En este modo de proceder el investigador dejó esos lugares de seguridad, otrora valorados, para abrirse a una experimentación que atravesó la propia subjetividad, de manera que se compusieron otras líneas en la experiencia interpretativa y en el rigor ético-estético. En este sentido la creación artística, en lugar de verse arrinconada por la investigación educativa, se valió de algunos de sus aspectos para encontrar otras vertientes de formalización inicialmente no contempladas.

Por otra parte, se comprendió que la gestualidad como experiencia corporal descentra la figura del profesor para aventurarse hacia una visión dramática del cuerpo en el contexto del aula escolar. Esto es, comprender los gestos en su dimensión performativa. Los cuerpos de los profesores se presentaron como espacio privilegiado para la construcción de saberes y la producción artística, sacándolos de ese rincón opaco al cual los ha reducido la didáctica, lo que condujo a reconocer que, más allá de los gestos corporales, habitan coreografías gestuales, inquietantes y perturbadoras. Las coreografías gestuales de los profesores son potentes y ampliamente polifónicas, sus sonoridades irrumpen de maneras inusitadas en la experiencia educativa y artística.

La producción estética construida en el contexto de esta investigación explora diversas vías de materialización de las coreografías gestuales, expandiendo con ello los bordes de comprensión del gesto corporal. Los materiales, las formas, las narraciones gestuales que configuran la obra artística, además de generar nuevas inquietudes con relación al perfomance de los profesores en el aula escolar, se convierten también en expresiones educativas y en re-construcciones de la fugacidad del gesto.

También es importante considerar que estos objetos (obra artística) guardan relación con la acción performativa del lenguaje sobre la materialidad; de manera que en los objetos están presentes las subjetividades de quienes los han producido, no solo en el sentido de una sensibilidad que se hace presente en el objeto, sino en una comprensión más amplia en la cual las 
subjetividades devienen objetos, objetualidades. Más aún, estos objetos constituyen formas de pensamiento; no representan un conocimiento externo a ellos, sino que en ellos el conocimiento aparece instaurado, desafiando los convencionalismos que reducen el conocimiento a un producto exclusivo de una organización racional, supeditada casi siempre al lenguaje escrito.

\section{Referencias}

Borgdorff, H. (2010).El debate sobre la investigación en las artes. Cairon: Revista de Ciencias de La Danza, (13), 25-46. Disponible en https://tinyurl.com/y48bdz8a

Finley, S. (2015). "Investigación con base en las artes" en N. K. Denzin \& Y. S. Lincoln (Ed.). Métodos de recolección y análisis de datos. Manual de investigación cualitativa. Volumen IV. (pp.113-139). Barcelona: Gedisa.

Hernández, E. (2015). “Cuerpos performados, cuerpos producidos: una reflexión teórica en perspectiva fenomenológica" en Temas Antropológicos, 37(1), 141-155.

Hernández, F. (2008). “La investigación basada en las artes. Propuestas para repensar lainvestigación en educación" en Educatio Siglo XXI, (26), 85-118. Disponible en https://goo.gl/eAJglo

Le Breton; David. (2000). El cuerpo y la educación en Revista Complutense, 11(2), 35-42. Disponible en https://goo.gl/Yys8Zn . (2002). Antropología del cuerpo y modernidad. Buenos Aires: Nueva Visión.
Marín, R. (2005). “La 'Investigación Educativa basada en las Artes Visuales' o 'Arteinvestigación Educativa'" en R. Marin Viandel (Coord.), Investigación en Educación Artística (pp. 223-274). Granada: Universidad de Granada.

(2011). “La Investigación en Educación

Artística" en Educatio Siglo XXI, 29(1), 211-230.

Disponible en https://goo.gl/yCk6XC

Meo-Zilio, G \& Mejía, S. (1983). Diccionario de Gestos: España e Hispanoamérica. Tomo I: A-H; Tomo II: I-Z. Instituto Caro y Cuervo: Bogotá.

Pallasmaa, J. (2006). Los ojos de la piel. Barcelona: Gustavo Gili.

Pedraza, Z. (2010). "Saber, cuerpo y escuela: El uso de los sentidos y la educación somática" en Calle14, 4(5), 43-57. Disponible en https://revistas.udistrital.edu.co/ojs/index.php/c14/ article/view/1202/1590

Preciado, B. (2002). Manifiesto contra-sexual. Madrid: Opera Prima.

Serres, M. (2011). Variaciones sobre el cuerpo. Buenos Aires: Fondo de Cultura Económica.

Scharagrodsky, P. (2001). El cuerpo en la escuela. Disponible en https://goo.gl/tv9xrK

Sibilia, P. (2005). El hombre postorgánico. Cuerpo, subjetividad y tecnologías digitales. Buenos Aires: Fondo de Cultura Económica. 